Commun. Fac. Sci. Univ. Ank. Ser. A1 Math. Stat.

Volume 69, Number 1, Pages 847-853(2020)

DOI: $10.31801 /$ cfsuasmas. 673191

ISSN 1303-5991 E-ISSN 2618-6470

http://communications.science.ankara.edu.tr

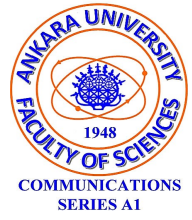

\title{
FOURIER-BESSEL TRANSFORMS OF DINI-LIPSCHITZ FUNCTIONS ON LEBESGUE SPACES $L_{p, \gamma}\left(\mathbb{R}_{+}^{n}\right)$
}

\author{
ISMAIL EKINCIOGLU, ESRA KAYA, AND S. ELIFNUR EKINCIOGLU
}

\begin{abstract}
In this paper, we prove a generalization of Titchmarsh's theorem for the Laplace-Bessel differential operator in the space $L_{p, \gamma}\left(\mathbb{R}_{+}^{n}\right)$ for functions satisfying the $(\psi, p)$-Laplace-Bessel Lipschitz condition for $1<p \leq 2$ and $\gamma>0$.
\end{abstract}

\section{INTRODUCTION}

Integral transforms and their inverse transforms are widely used to solve various problems in calculus, fourier analysis, mechanics, mathematical physics, and computational mathematics. Fourier transform is one of the most important integral transforms. Since it was introducted by Fourier in the early 1880s, it has become an important mathematical concept that is at the centre of the highly developed branch of mathematics called Fourier Analysis. It has many application areas. The Fourier transform of the kernel of singular integral operator is very important in applications of singular integral operator theory. The properties of the Fourier transform of the kernel give information about the existence of the solution of singular integral equations. Since singular integrals are convolution type operators, their Fourier transforms are the product of the Fourier transforms of two functions.

As it is well known that if Lipschitz conditions are applied on a function $f(x)$, then these conditions greatly affect the absolute convergence of the Fourier-Bessel series and behaviour of $F_{\gamma} f$ Fourier-Bessel transforms of $f$. In general, if $f(x)$ belongs to a certain function class, then the Lipschitz conditions have bearing as to the dual space to which the Fourier coefficients and Fourier-Bessel transforms of $f(x)$ belong. Younis (see 12]) worked the same phenomena for the wider Dini Lipschitz class for some classes of functions. Daher, El Quadih, Daher and El Hamma proved an analog Younis (see [12, Theorem 2.5]) in for the Fourier-Bessel transform for functions satisfies the Fourier-Bessel Dini Lipschitz condition in the

Received by the editors: January 10, 2020; Accepted: March 06, 2020.

2010 Mathematics Subject Classification. Primary 42B10; Secondary 26A16.

Key words and phrases. Laplace-Bessel differential operator, generalized shift operator, Laplace-Bessel Lipschitz function.

(C)2020 Ankara University Communications Faculty of Sciences University of Ankara-Series A1 Mathematics and Statistics 
Lebesgue space $L_{\alpha, n}^{2}$ (see [10]). El Hamma and Daher proved a generalization of Titchmarsh's theorem for the Bessel transform in the space $L_{2, \gamma}\left(\mathbb{R}_{+}^{n}\right)$ (see [1] ) .

In this paper we prove a generalization of Titchmarsh's theorem for the LaplaceBessel transform in the space $L_{p, \gamma}\left(\mathbb{R}_{+}^{n}\right)$, where $1<p \leq 2$ and $\gamma>0$.

\section{Preliminaries}

Let $\mathbb{R}_{+}^{n}$ be the part of the Euclidean space $\mathbb{R}^{n}$ of points $x=\left(x_{1}, \ldots, x_{n}\right)$, defined by the inequality $x_{n}>0$. We write $x=\left(x^{\prime}, x_{n}\right), x^{\prime}=\left(x_{1}, \ldots, x_{n-1}\right) \in \mathbb{R}_{+}^{n-1}$. $S_{+}^{n}$ denote the unit sphere on $\mathbb{R}_{+}^{n}$, which can be defined as $S_{+}^{n}=\left\{x \in \mathbb{R}_{+}^{n}:|x|=1\right\}$. $\mathbb{S}_{+}=\mathbb{S}\left(\mathbb{R}_{+}^{n}\right)$ be the space of functions which are the restrictions to $\mathbb{R}_{+}^{n}$ of the test functions of the Schwartz that are even with respect to $x_{n}$, decreasing sufficiently rapidly at infinity, together with all derivatives of the form

$$
D_{\gamma}^{\alpha}=D_{x^{\prime}}^{\alpha^{\prime}} B_{n}^{\alpha_{n}}=D_{1}^{\alpha_{1}} \ldots D_{n-1}^{\alpha_{n-1}} B_{n}^{\alpha_{n}}=\frac{\partial^{\alpha_{1}}}{\partial x_{1}^{\alpha_{1}}} \ldots \ldots \frac{\partial^{\alpha_{n-1}}}{\partial x_{n-1}^{\alpha_{n-1}}} B_{n}^{\alpha_{n}},
$$

i.e., for all $\varphi \in \mathbb{S}_{+}, \sup _{x \in \mathbb{R}_{+}^{n}}\left|x^{\beta} D_{\gamma}^{\alpha} \varphi\right|<\infty$, where $\alpha=\left(\alpha_{1}, \ldots, \alpha_{n}\right)$ and $\beta=\left(\beta_{1}, \ldots, \beta_{n}\right)$ are multi-indexes, and $x^{\beta}=x_{1}^{\beta_{1}} \ldots x_{n}^{\beta_{n}}$ and $B_{n}=\frac{\partial^{2}}{\partial x_{n}^{2}}+\frac{\gamma}{x_{n}} \frac{\partial}{\partial x_{n}}$ is the Bessel differential expansion. For $\gamma \geq 0$, we introduce the Bessel normalized function of the first kind $j_{\gamma}$ defined by

$$
j_{\gamma}(z)=\Gamma(\gamma+1) \sum_{n=0}^{\infty} \frac{(-1)^{n}}{n ! \Gamma(n+\gamma+1)}\left(\frac{z}{2}\right)^{2 n}
$$

where $\Gamma$ is the gamma-function (see [9]). Moreover, from (1) we see that

$$
\lim _{z \rightarrow 0} \frac{j_{\frac{\gamma-1}{2}}(z)-1}{z^{2}} \neq 0
$$

by consequence, there exist $C>0$ and $\eta>0$ satisfying

$$
|z| \leq \eta \Rightarrow\left|j_{\frac{\gamma-1}{2}}(z)-1\right| \geq C|z|^{2}
$$

The function $u=j_{\frac{\gamma-1}{2}}(z)$ satisfies the differential equation

$$
B_{x_{n}} u(x, y)=B_{y_{n}} u(x, y)
$$

with the initial conditions $u(x, 0)=f(x)$ and $u_{y}(x, 0)=0$ is function infinitely differentiable, even, and, moreover entire analytic.

The Fourier-Bessel transformation and its inverse on $\mathbb{S}_{+}$are defined by

$$
\begin{aligned}
F_{\gamma} f(x) & =\int_{\mathbb{R}^{n}} f(y) e^{-i\left(x^{\prime} y^{\prime}\right)} j_{\frac{\gamma-1}{2}}\left(x_{n} y_{n}\right) y_{n}^{\gamma} d y, \\
F_{\gamma}^{-1} f(x) & =C_{n, \gamma} F_{\gamma} f\left(-x^{\prime}, x_{n}\right),
\end{aligned}
$$


where $\left(x^{\prime}, y^{\prime}\right)=x_{1} y_{1}+\ldots+x_{n-1} y_{n-1}, j_{\gamma}, \gamma>0$, is the normalized Bessel function, and

$$
C_{n, \gamma}=(2 \pi)^{n-1} 2^{\gamma-1} \Gamma^{2}((\gamma+1) / 2),
$$

(see [4, 9, 11]). This transform is associated to the Laplace-Bessel differential operator

$$
\Delta_{\gamma}=\sum_{i=1}^{n} \frac{\partial^{2}}{\partial x_{i}^{2}}+\frac{\gamma}{x_{n}} \frac{\partial}{\partial x_{n}}, \gamma>0
$$

The expression (3) is a hybrid of the Hankel transform.

For a fixed parameter $\gamma>0$, let $L_{p, \gamma}=L_{p, \gamma}\left(\mathbb{R}_{+}^{n}\right)$ be the space of measurable functions with a finite norm

$$
\|f\|_{L_{p, \gamma}}=\left(\int_{\mathbb{R}_{+}^{n}}|f(x)|^{p} x_{n}^{\gamma} d x\right)^{1 / p}, \quad 1 \leq p<\infty .
$$

The space of the essentially bounded measurable function on $\mathbb{R}_{+}^{n}$ is denoted by $L_{\infty, \gamma}\left(\mathbb{R}_{+}^{n}\right)$. For for $f \in L_{p, \gamma}$, I.A. Kipriyanov (for $n=1$ B.M. Levitan [7, 8]) investigated the generalized convolution $\left(\Delta_{\gamma}\right.$-convolution $)$

$$
(f \otimes g)(x)=\int_{\mathbb{R}_{+}^{n}} f(y) T^{y} g(x) y_{n}^{\gamma} d y,
$$

associated with the Laplace-Bessel differential operator, where $T^{y}$ is the generalized shift operator $\left(\Delta_{\gamma}\right.$-shift $)$ defined by

$$
T^{y} f(x)=C_{\gamma} \int_{0}^{\pi} f\left(x^{\prime}-y^{\prime}, \sqrt{x_{n}^{2}-2 x_{n} y_{n} \cos \theta+y_{n}^{2}}\right) \sin ^{\gamma-1} \theta d \theta
$$

being $C_{\gamma}=\pi^{-\frac{1}{2}} \Gamma\left(\frac{\gamma+1}{2}\right)\left[\Gamma\left(\frac{\gamma}{2}\right)\right]^{-1}$ (see [5, 6, 7, 8]). We note that this convolution satisfies the property $(f \otimes g)(x)=(g \otimes f)(x)$ (see [2, 3]). The following relation connect the generalized shift operator and the Fourier-Bessel transform, we have

$$
F_{\gamma}\left[T^{y} f(x)\right]=j_{\frac{\gamma-1}{2}}\left(x_{n} y_{n}\right) F_{\gamma}[f(x)] .
$$

Given $1<p \leq 2, \frac{1}{p}+\frac{1}{q}=1$ and $f \in L_{p, \gamma}$, we have the Hausdorff-Young inequality

$$
\left\|F_{\gamma} f\right\|_{q, \gamma} \leq C_{q}\|f\|_{p, \gamma},
$$

where and $C_{q}$ is a positive constant.

\section{Fourier-Bessel Transforms of Dini-Lipschitz Functions}

In this section we give the main result of this paper. We need first to define $(\psi, p)$-Laplace Bessel Lipschitz class.

Definition 1. A function $f \in L_{p, \gamma}\left(\mathbb{R}_{+}^{n}\right)$ is said to be in the $(\psi, p)$-Laplace Bessel Lipschitz class, denoted by $\operatorname{Lip}(\psi, \gamma, p)$, if

$$
\left\|T^{y} f(x)-f(x)\right\|_{p, \gamma}=O(\psi(y)) \quad \text { as } \quad y \rightarrow 0
$$


where $\psi(x)$ is a continuous increasing function on $\mathbb{R}_{+}^{n}, \psi(0)=0$, and $\psi(x s)=$ $\psi(x) \psi(s)$ for all $x, s \in \mathbb{R}_{+}^{n}$.

Theorem 2. Let $f(x)$ belong to $\operatorname{Lip}(\psi, \gamma, p)$. Then

$$
\int_{|\xi| \geq \tau}\left|F_{\gamma} f(\xi)\right|{ }^{q} \xi_{n}^{\gamma} d \xi=O\left(\psi\left(\tau^{-q}\right)\right), \quad \text { as } \quad \tau \rightarrow+\infty .
$$

Proof. Let $f \in \operatorname{Lip}(\psi, \gamma, p)$. Then we have

$$
\left\|T^{y} f(x)-f(x)\right\|_{p, \gamma}=O(\psi(y)) \quad \text { as } \quad y \rightarrow 0 .
$$

Now we consider Fourier-Bessel transform of generalized shift operator. We get

$$
\begin{aligned}
F_{\gamma}\left[T^{y} f(x)\right](\xi) & =\int_{\mathbb{R}_{+}^{n}} T^{y} f(x) j_{\frac{\gamma-1}{2}}\left(x_{n} \xi_{n}\right) x_{n}^{\gamma} d x \\
& =\int_{\mathbb{R}_{+}^{n}} T^{y}\left[j_{\frac{\gamma-1}{2}}\left(x_{n} \xi_{n}\right)\right] f(x) x_{n}^{\gamma} d x \\
& =\int_{\mathbb{R}_{+}^{n}} j_{\frac{\gamma-1}{2}}\left(x_{n} \xi_{n}\right) j_{\frac{\gamma-1}{2}}\left(y_{n} \xi_{n}\right) f(x) x_{n}^{\gamma} d x \\
& =j_{\frac{\gamma-1}{2}}\left(y_{n} \xi_{n}\right) \int_{\mathbb{R}_{n}^{+}} f(x) j_{\frac{\gamma-1}{2}}\left(x_{n} \xi_{n}\right) x_{n}^{\gamma} d x \\
& =j_{\frac{\gamma-1}{2}}\left(y_{n} \xi_{n}\right) F_{\gamma}(f)(\xi),
\end{aligned}
$$

where $T^{y}\left(j_{p}(\sqrt{\lambda} x)\right)=j_{p}(\sqrt{\lambda} y) j_{p}(\sqrt{\lambda} x)$. From formulas 44 and $[5$, we obtain

$$
\begin{aligned}
\int_{\mathbb{R}_{+}^{n}} F_{\gamma}\left|T^{y} f(x)-f(x)\right|^{q} x_{n}^{\gamma} d x & =\int_{\mathbb{R}_{+}^{n}}\left|F_{\gamma} T^{y} f(x)-F_{\gamma} f(x)\right|^{q} x_{n}^{\gamma} d x \\
& =\int_{\mathbb{R}_{+}^{n}}\left|j_{\frac{\gamma-1}{2}}(\xi y) F_{\gamma} f(\xi)-F_{\gamma} f(\xi)\right|^{q} \xi_{n}^{\gamma} d \xi \\
& =\int_{\mathbb{R}_{+}^{n}}\left|F_{\gamma} f(\xi)\left[1-j_{\frac{\gamma-1}{2}}(\xi y)\right]\right|^{q} \xi_{n}^{\gamma} d \xi \\
& =\int_{\mathbb{R}_{+}^{n}}\left|1-j_{\frac{\gamma-1}{2}}(\xi y)\right|^{q}\left|F_{\gamma} f(\xi)\right|^{q} \xi_{n}^{\gamma} d \xi \\
& \leq C_{q} \int_{\mathbb{R}_{+}^{n}}\left|T^{y} f(x)-f(x)\right|^{q} \xi_{n}^{\gamma} d \xi \\
& \leq C_{q}\left\|T^{y} f(x)-f(x)\right\|_{p, \gamma}^{q} .
\end{aligned}
$$

From (2), we have

$$
\begin{aligned}
\int_{\frac{1}{h} \leq|\xi| \leq \frac{2}{h}}\left|F_{\gamma} f(\xi)\right|^{q} \xi_{n}^{\gamma} d \xi & =C_{q} \int_{\frac{1}{h} \leq|\xi| \leq \frac{2}{h}}\left|1-j_{\frac{\gamma-1}{2}}(\xi h)\right|^{q}\left|F_{\gamma} f(\xi)\right|^{q} \xi_{n}^{\gamma} d \xi \\
& \geq C_{q}|h|^{-1} \int_{\frac{1}{h} \leq|\xi| \leq \frac{2}{h}}\left|F_{\gamma} f(\xi)\right|^{q} \xi_{n}^{\gamma} d \xi
\end{aligned}
$$


$0<h \leq 1$. It follows from the above consideration that there exists a positive constant $C$ such that

$$
\int_{\frac{1}{h} \leq|\xi| \leq \frac{2}{h}}\left|F_{\gamma} f(\xi)\right|^{q} \xi_{n}^{\gamma} d \xi \leq C \psi^{q}(h)=C \psi\left(h^{q}\right) .
$$

Therefore, we get

$$
\int_{\tau \leq|\xi| \leq 2 \tau}\left|F_{\gamma} f(\xi)\right|^{q} \xi_{n}^{\gamma} d \xi \leq C \psi\left(\tau^{-q}\right)
$$

In fact, we have

$$
\begin{aligned}
\int_{\tau \leq|\xi|<\infty}\left|F_{\gamma} f(\xi)\right|^{q} \xi_{n}^{\gamma} d \xi & =\sum_{k=1}^{\infty} \int_{2^{k-1} \tau \leq|\xi|<2^{k} \tau}\left|F_{\gamma} f(\xi)\right|^{q} \xi_{n}^{\gamma} d \xi \\
& \leq C_{q} \psi\left(\tau^{-q}\right)+C_{q} \psi\left((2 \tau)^{-q}\right)+C_{q} \psi\left(\left(2^{2} \tau\right)^{-q}\right)+\ldots \\
& \leq C_{q} \psi\left(\tau^{-q}\right)\left(1+\psi\left(2^{-q}\right)+\psi^{2}\left(2^{-q}\right)+\psi^{3}\left(2^{-q}\right)+\ldots\right) .
\end{aligned}
$$

Thus, we can write

$$
\int_{\tau \leq|\xi|<\infty}\left|F_{\gamma} f(\xi)\right|^{q} \xi_{n}^{\gamma} d \xi \leq C_{1} \psi\left(\tau^{-q}\right),
$$

where $C_{1}=C_{q}\left(1-\psi\left(2^{-q}\right)\right)^{-1}$ since $2^{-q}<1$. Finally, we get

$$
\int_{|\xi| \geq \tau}\left|F_{\gamma} f(\xi)\right|^{q} \xi_{n}^{\gamma} d \xi=O\left(\psi\left(\tau^{-q}\right)\right) \quad \text { as } \quad \tau \rightarrow \infty .
$$

Thus, the proof of theorem is completed.

We can give the following result which is used for many the theorem given above. It is well known that

$$
\begin{gathered}
F_{\gamma}\left(B_{n}^{\alpha_{n}} f\right)(x)=\left(-x_{n}^{2}\right)^{\alpha_{n}} F_{\gamma} f(x), \\
F_{\gamma}\left(D_{i}^{2 \alpha_{i}} f\right)(x)=\left(-x_{i}^{2}\right)^{\alpha_{i}} F_{\gamma} f(x), \quad i=1, \ldots, n-1, \\
F_{\gamma}\left(\Delta_{\gamma} f\right)(x)=-|x|^{2} F_{\gamma} f(x) \text { and } F_{\gamma}(f \otimes g)=F_{\gamma} f F_{\gamma} g, \\
F_{\gamma}\left(D_{x^{\prime}}^{2 \alpha^{\prime}} B_{n}^{\alpha_{n}} f\right)(x)=(-1)^{|\alpha|} x^{2 \alpha} F_{\gamma} f(x)
\end{gathered}
$$

We can use the mathematical induction method for $k=1$, we get

$$
\begin{aligned}
F_{\gamma}\left(\Delta_{\gamma} f\right)(x) & =C_{n, \gamma} \int_{\mathbb{R}_{+}^{n}} \Delta_{\gamma} f(y) e^{-i x^{\prime} y^{\prime}} j_{\frac{\gamma-1}{2}}\left(x_{n} y_{n}\right) y_{n}^{\gamma} d y \\
& =C_{n, \gamma} \int_{\mathbb{R}_{+}^{n}}\left(\sum_{k=1}^{n} \frac{\partial^{2} f(y)}{\partial y_{k}^{2}}+\frac{\gamma}{y_{n}} \frac{\partial f(y)}{\partial y_{n}}\right) e^{-i x^{\prime} y^{\prime}} j_{\frac{\gamma-1}{2}}\left(x_{n} y_{n}\right) y_{n}^{\gamma} d y
\end{aligned}
$$




$$
\begin{aligned}
& =C_{n, \gamma} \int_{\mathbb{R}_{+}^{n}}\left(\sum_{k=1}^{n} \frac{\partial^{2} f(y)}{\partial y_{k}^{2}} e^{-i x^{\prime} y^{\prime}} j_{\frac{\gamma-1}{2}}\left(x_{n} y_{n}\right) y_{n}^{\gamma} d y\right. \\
& -C_{n, \gamma} \int_{\mathbb{R}_{+}^{n}}\left(\sum_{k=1}^{n} \frac{\gamma}{y_{n}} \frac{\partial f(y)}{\partial y_{n}}\right) e^{-i x^{\prime} y^{\prime}} j_{\frac{\gamma-1}{2}}\left(x_{n} y_{n}\right) y_{n}^{\gamma} d y=I_{1}+I_{2} .
\end{aligned}
$$

If we apply partial integration to the second term of $I_{1}$ and $I_{2}$, then we have

$$
F_{\gamma}\left(\Delta_{\gamma} u\right)(x)=C_{n, \gamma} \int_{\mathbb{R}_{+}^{n}} f(y) e^{-i x^{\prime} y^{\prime}}\left(\Delta_{\gamma} j_{\frac{\gamma-1}{2}}\left(x_{n} y_{n}\right)\right) y_{n}^{\gamma} d y .
$$

Here, if we use the following equality $[8$,

$$
\int_{0}^{\infty} f(y) \Delta_{\gamma} j_{\frac{\gamma-1}{2}}(x y) y^{\gamma} d y=-|x|^{2} \int_{0}^{\infty} f(y) j_{\frac{\gamma-1}{2}}(x y) y^{\gamma} d y
$$

then we have

$$
F_{\gamma}\left(\Delta_{\gamma} f\right)(x)=-|x|^{2} F_{\gamma} f(x) .
$$

Since $f \in \operatorname{Lip}(\psi, \gamma, p)$, it is clear that

$$
\left\|F_{\gamma}\left(\Delta_{\gamma} f\right)\right\|_{L_{q, \gamma}(|\xi| \geq \tau)} \leq C_{n, \gamma} O\left(\psi\left(\tau^{-q}\right)\right)
$$

as $\tau \rightarrow+\infty$.

There are many examples. Here is one of them and a simple method to produce many more: $f(x)=|x|^{\frac{1}{p}}$ for $1<p<\infty$, where $f(0)=0$ is understood. These functions are uniformly continuous on all of $\mathbb{R}_{+}^{n}$. If $p=2, f$ belongs to the Lipschitz class at $\mathbb{R}_{+}$.

\section{REFERENCES}

[1] El Hamma, M. and Daher, R., Generalization of Titchmarsh's Theorem for the Bessel transform, Rom. J. Math. Comput. Sci. 2(2), (2012), 17-22.

[2] Ekincioglu, I. and Ozkin, I. K., On high order Riesz transformations generated by a generalized shift operator, Tr. J. Math. 21 (1997), 51-60.

[3] Ekincioglu, I. and Serbetci, A., On the singular integral operators generated by the generalized shift operator, Int. J. App. Math. 1 (1999), 29-38.

[4] Kipriyanov, I. A., Singular Elliptic Boundary Value Problems, Nauka, Moscow, Russia, 1997.

[5] Kipriyanov, I. A. and Lyakhov, L. N., Multipliers of the mixed Fourier-Bessel transform, Dokl. Akad. Nauk. 354(4), (1997), 449-451.

[6] Kipriyanov, I. A. and Klyuchantsev, M. I., On Singular Integrals Generated by The Generalized Shift Operator, II. Sib. Mat. Zh. 11 (1970), 1060-1083.

[7] Levitan, B. M., The Theory of Generalized Translation Operators, Nauka, Moscow, Russia, 1973.

[8] Levitan, B. M., Bessel function expansions in series and Fourier integrals, Uspekhi Mat. Nauk 6. 42(2), (1951), 102-143.

[9] Levitan, B. M., Expansion in Fourier series and integrals over Bessel functions, Uspekhi Mat. Nauk 6. 2 (1951), 102-143.

[10] El Quadih, S., Daher, R. and El Hamma, M., Generalization of Titchmarsh's Theorem for the Generalized Fourier-Bessel Transform in the Space $L_{\alpha, n}^{2}$, International Journal of Mathematical Modelling Computations 6(3), (2016), 253-260. 
[11] Trimèche, K., Transmutation operators and mean-periodic functions associated with differential operators, Math. Rep. 4(1), (1988), 1-282.

[12] Younis, M. S., Fourier transforms of Dini-Lipschitz functions, Internat. J. Math. E Math. Sci. 9(2), (1986) 301-312.

Current address: Ismail Ekincioglu: Department of Mathematics, Dumlupinar University, Kutahya, Turkey.

E-mail address: ismail.ekincioglu@dpu.edu.tr

ORCID Address: https://orcid.org/0000-0002-5636-1214

Current address: Esra Kaya: Department of Mathematics, Dumlupinar University, Kutahya, Turkey.

E-mail address: kayaesra.e.k@gmail.com

ORCID Address: https://orcid.org/0000-0002-6971-0452

Current address: S. Elifnur Ekincioglu: Department of Mathematics, Dumlupinar University, Kutahya, Turkey.

E-mail address: ekinciogluelifnur@gmail.com

ORCID Address: https://orcid.org/0000-0001-6700-8232 